PROCEEDINGS OF THE

AMERICAN MATHEMATICAL SOCIETY

Volume 131, Number 1, Pages 219-229

S 0002-9939(02)06555-3

Article electronically published on May 1, 2002

\title{
NECESSARY AND SUFFICIENT CONDITIONS FOR ABSOLUTE SUMMABILITY OF THE TRACE FORMULAS FOR CERTAIN ONE DIMENSIONAL SCHRÖDINGER OPERATORS
}

\author{
ALEXEI RYBKIN \\ (Communicated by Carmen C. Chicone)
}

\begin{abstract}
For the general one dimensional Schrödinger operator $-\frac{d^{2}}{d x^{2}}+q(x)$ with real $q$ we study some analytic aspects related to order-one trace formulas originally due to Buslaev-Faddeev, Faddeev-Zakharov, and Gesztesy-HoldenSimon-Zhao. We show that the condition $q \in L_{1}(\mathbb{R})$ guarantees the existence of the trace formulas of order one only with certain resolvent regularizations of the integrals involved. Our principle results are simple necessary and sufficient conditions on absolute summability of the formulas under consideration. These conditions are expressed in terms of Fourier transforms related to $q$.
\end{abstract}

\section{IntRoduction}

We will consider differential operations of the form

$$
l \equiv-\frac{d^{2}}{d x^{2}}+q(x)
$$

with real potentials in half-line and full-line cases.

Trace formulas for various Schrödinger operators associated with $l$ have been intensively studied since the pioneering work [6] by Gel'fand, Levitan and [4 by Dikii. Leaving aside numerous papers devoted to this area we mention only [3], [5], [7]-[11, [15], [16], which we will be dealing with here. The interested reader can find in these further references. In general, trace formulas we are concerned with are chains of relations linking regularized moments of certain spectral/scattering characteristics (e.g. the so-called spectral shift function $\xi$, see below) with some characteristics of $q$ 's. Most of the earlier work and work done by physicists is either formal or stated under excessively strong conditions on potentials. It is clear that the actual number of such formulas depends on the smoothness of $q$ 's. But this fact appears not to have been expressed in exact terms. It is even natural to ask a simpler question. Given a Schrödinger operator $H$ defined by (1.1) with a potential $q$ subject to an optimal condition providing existence of certain scattering (spectral) characteristics of $H$, how many trace formulas can one derive and how many of those are absolutely summable? Some related questions were recently treated in [10], [16], [21] but we can find only partial answers.

Received by the editors May 8, 2001 and, in revised form, August 31, 2001.

1991 Mathematics Subject Classification. Primary 34L40, 47E05; Secondary 34E40.

Key words and phrases. Schrödinger operator, Krein's spectral shift function, trace formula.

(C)2002 American Mathematical Society 
The goal of the present note is to give a complete answer to this question in three important cases: full-line with arbitrary $q$ (Theorem 2.4), full-line with summable $q$ (Theorem 3.2), and half-line with summable $q$ (Theorem 4.1). The exposition is based upon a recent result [17] on suitable estimates of the error terms in asymptotic representations of the Jost solution and Weyl $m$-function. Some other trace formulas as well as higher order relations will be discussed in detail in [18.

Notation. $\mathbb{R}_{ \pm}=( \pm \infty, 0), \mathbb{C}_{ \pm}=\{z \in \mathbb{C}: \pm \operatorname{Im} z>0\}, \overline{\mathbb{C}}_{ \pm}=\mathbb{C}_{ \pm} \cup \mathbb{R}$. We will also use standard function spaces $(1 \leq p<\infty)$

$$
\begin{aligned}
& L_{p}(\Delta, d \mu)=\left\{f:\|f\|_{p}^{p} \equiv \int_{\Delta}|f(x)|^{p} d \mu<\infty\right\}, L_{p}(\Delta, d x) \equiv L_{p}(\Delta), \\
& L_{\infty}(\Delta)=\left\{f:\|f\|_{\infty} \equiv \text { ess } \sup _{x \in \Delta}|f(x)|<\infty\right\}, L_{p, \text { loc }}=\left\{\cap L_{p}(\Delta): \Delta \text { is compact }\right\}
\end{aligned}
$$

and $H^{n, p}(\Delta)$ denotes the usual Sobolev space whose elements have up to $n$ distributional derivatives in $L_{p}(\Delta)$.

Given the self-adjoint operator $H, \sigma_{d}(H), \sigma_{s c}(H)$, and $\sigma_{a c}(H)$ are, respectively, the discrete, singular continuous, and absolutely continuous spectrum of $H$.

Krein's spectral shift function. Let $\left(H, H_{0}\right)$ be an abstract pair of resolvent comparable Hilbert space operators (i.e. $(H-z I)^{-1}-\left(H_{0}-z I\right)^{-1}$ is trace class for a complex $z$ ). Then (see, e.g. [2]) there exists a unique (up to an additive constant) real-valued function $\xi \in L_{1}\left(\mathbb{R}, \frac{d t}{1+t^{2}}\right)$ such that the Krein trace formula

$$
\operatorname{tr}\left\{\varphi(H)-\varphi\left(H_{0}\right)\right\}=\int_{\mathbb{R}} \varphi^{\prime}(t) \xi(t) d t
$$

holds, $\varphi$ being an arbitrary function of some suitable class. The function $\xi$ is called the Krein spectral shift function of $\left(H, H_{0}\right)$.

\section{Full Line CASE With ARBitrary POTENTIALS}

In this section we consider a pair of operators $\left(H, H_{x_{0}}\right)$ defined on $L_{2}(\mathbb{R})$ by (1.1):

$$
\begin{aligned}
H u & =l u, u \in \operatorname{Dom}(H)=\left\{f \in L_{2}(\mathbb{R}): l f \in L_{2}(\mathbb{R})\right\}, \\
H_{x_{0}}^{ \pm} u & =l u, u \in \operatorname{Dom}\left(H_{x_{0}}^{ \pm}\right) \\
& =\left\{f \in L_{2}(x, \pm \infty): l f \in L_{2}\left(x_{0}, \pm \infty\right), f\left(x_{0} \pm 0\right)=0\right\}, \\
H_{x_{0}} & =H_{x_{0}}^{+} \oplus H_{x_{0}}^{-} .
\end{aligned}
$$

If $q$ is in the so-called limit circle case at $\pm \infty$ (see, e.g. [19]), then operators in (2.1)(2.3) are no longer well-defined. In this case one has to impose some selfadjoint boundary conditions at $\pm \infty$. For simplicity, we assume that our $q$ 's are in the limit point (l.p.) case at $\pm \infty$, i.e. (2.1)-(2.3) define selfadjoint operators.

Since $\left(H_{x_{0}}-i\right)^{-1}-(H-i)^{-1}$ is rank one (see, e.g. [11]), the spectral shift function $\xi\left(x_{0}, t\right)$ of $\left(H, H_{x_{0}}\right)$ exists.

The following remarkable trace formula was discovered by Gesztesy, Holden, Simon, and Zhao in [7] (see also [9], [10], [11]). In the present form it was proven in [16]. 
Proposition 2.1. Let $q \in L_{1, l o c}$ and in l.p. case at $\pm \infty$ and let $\xi\left(x_{0}, t\right)$ be the spectral shift function of $\left(H, H_{x_{0}}\right)$. Then for almost all $x_{0} \in \mathbb{R}$

$$
\frac{q\left(x_{0}-0\right)+q\left(x_{0}+0\right)}{2}=\lim _{\varepsilon \rightarrow+0} \int_{\mathbb{R}} \frac{1}{(1+i \varepsilon t)^{2}}\left\{\chi(t)-2 \xi\left(x_{0}, t\right)\right\} d t,
$$

where $\chi$ is the Heaviside function.

Remark 2.2. Formula (2.4) is the first one in a chain of higher order trace relations 9]. It was demonstrated in [10] that, in general, the resolvent regularization in (2.4) cannot be removed. This means that there are no absolutely summable trace formulas in this setting.

Our main concern in this section will be conditions on $q$ 's providing absolute summability of the right-hand side of (2.4). Our study of $\xi\left(x_{0}, t\right)$ will be based upon Titchmarsh-Weyl's $m$-function. We briefly recall its definition. According to the Weyl theory (see, e.g. [12], [19]) for every real potential $q \in L_{1, \text { loc }}$ subject to the limit point case at $+\infty$ the equation

$$
-u^{\prime \prime}+q(x) u=\lambda u, x \in\left(x_{0}, \infty\right)
$$

has a solution $u(x, \lambda)$ belonging to $L_{2}\left(x_{0}, \infty\right)$ for all $\lambda \in \mathbb{C}_{+}$. The Titchmarsh-Weyl $m$-function associated with $H_{x_{0}}^{+}$is then defined as

$$
m_{+}\left(x_{0}, \lambda\right)=\frac{u^{\prime}\left(x_{0}+0, \lambda\right)}{u\left(x_{0}+0, \lambda\right)} .
$$

The function $m_{+}\left(x_{0}, \lambda\right)$ is unique, analytic in $\lambda \in \mathbb{C} \backslash \sigma\left(H_{x_{0}}^{+}\right)$, and maps $\mathbb{C}_{+}$into itself. In a similar manner, one defines $m_{-}\left(x_{0}, \lambda\right)$ corresponding to $H_{x_{0}}^{-}$which maps $\mathbb{C}_{-}$into itself. The following statement [17] is a refinement of one statement due to Atkinson [1]. We believe it can also be derived from [12].

Proposition 2.3. Let $q \in L_{1}(\mathbb{R})$. Then

$$
m_{ \pm}\left(x_{0}, \lambda\right)= \pm i \sqrt{\lambda}-\int_{x_{0}}^{ \pm \infty} e^{ \pm 2 i \sqrt{\lambda}\left(x-x_{0}\right)} q(x) d x+r_{ \pm}\left(x_{0} ; \lambda\right)
$$

where $\left\|r_{ \pm}(\cdot ; \lambda)\right\|_{\infty}=O\left(|\lambda|^{-1 / 2}\right)$ and $r_{ \pm}\left(x_{0} ; \lambda\right)=o\left(|\lambda|^{-1 / 2}\right), \forall x_{0} \in \mathbb{R}$, uniformly in $\overline{\mathbb{C}}_{+}, \lambda \rightarrow \infty$.

We now state and prove the main result of this section.

Theorem 2.4. If $q \in L_{1}(\mathbb{R})$, then (2.4) is absolutely summable if and only if

$$
\int_{\mathbb{R}} q(x) \cos t\left(x-x_{0}\right) d x \in L_{1}\left(\mathbb{R}_{+}, d t\right) .
$$

Formula (2.4) then reads

$$
\frac{q\left(x_{0}-0\right)+q\left(x_{0}+0\right)}{2}=E+\int_{E}^{\infty}\left\{1-2 \xi\left(x_{0}, t\right)\right\} d t, E=\inf \sigma(H) .
$$

Condition (2.5) holds if $q \in H^{1,1}(\mathbb{R}) \cap H^{1,2}(\mathbb{R})$.

Proof. Note first that under our condition $q \in L_{1}(\mathbb{R})$ the spectrum of $H$ is bounded below by $E=\inf \sigma(H)$ and (2.4) then reads

$$
\frac{q\left(x_{0}-0\right)+q\left(x_{0}+0\right)}{2}=E+\lim _{\varepsilon \rightarrow 0} \int_{E}^{\infty} \frac{1}{(1+i \varepsilon t)^{2}}\left\{1-2 \xi\left(x_{0}, t\right)\right\} d t .
$$


We use the following representation [11]:

$$
1-2 \xi\left(x_{0}, t\right)=\frac{1}{\pi} \operatorname{Im} \ln \frac{m_{+}\left(x_{0}, t+i 0\right)-m_{-}\left(x_{0}, t-i 0\right)}{i \sqrt{t}} .
$$

By Proposition 2.3 we have

$$
\frac{m_{+}\left(x_{0}, t+i 0\right)-m_{-}\left(x_{0}, t-i 0\right)}{i \sqrt{t}}=2\left\{1-\frac{1}{2 i \sqrt{t}} \widetilde{q}\left(x_{0}, t\right)\right\}+O\left(\frac{1}{t^{3 / 2}}\right), t \rightarrow \infty,
$$

where

$$
\widetilde{q}\left(x_{0}, t\right)=\int_{-\infty}^{x_{0}} e^{2 i \sqrt{t}\left(x_{0}-x\right)} q(x) d x+\int_{x_{0}}^{\infty} e^{2 i \sqrt{t}\left(x-x_{0}\right)} q(x) d x,
$$

and (2.8) continues:

$$
\begin{aligned}
1-2 \xi\left(x_{0}, t\right) & =\frac{1}{2 \pi \sqrt{t}}\left\{\operatorname{Re} \widetilde{q}\left(x_{0}, t\right)-\frac{1}{2 \sqrt{t}} \operatorname{Im} \widetilde{q}^{2}\left(x_{0}, t\right)\right\}+O\left(\frac{1}{t^{3 / 2}}\right) \\
& =\frac{\operatorname{Re} \widetilde{q}\left(x_{0}, t\right)}{2 \pi \sqrt{t}}\left\{1-\frac{1}{\sqrt{t}} \operatorname{Im} \widetilde{q}\left(x_{0}, t\right)\right\}+O\left(\frac{1}{t^{3 / 2}}\right), t \rightarrow \infty .
\end{aligned}
$$

Since $\left\|\widetilde{q}\left(x_{0}, \cdot\right)\right\|_{\infty} \leq\|q\|_{1}$ we have

$$
1-\frac{1}{\sqrt{t}}\|q\|_{1} \leq\left|1-\frac{1}{\sqrt{t}} \operatorname{Im} \widetilde{q}\left(x_{0}, t\right)\right| \leq 1+\frac{1}{\sqrt{t}}\|q\|_{1},
$$

which implies that there is $a>0$ :

$$
C_{1}\left\|\widetilde{q}\left(x_{0}, \cdot\right)\right\|_{1} \leq \int_{a}^{\infty}\left|\frac{\operatorname{Re} \widetilde{q}\left(x_{0}, t\right)}{\sqrt{t}}\left\{1-\frac{1}{\sqrt{t}} \operatorname{Im} \widetilde{q}\left(x_{0}, t\right)\right\}\right| d t \leq C_{2}\left\|\widetilde{q}\left(x_{0}, \cdot\right)\right\|_{1}
$$

with some $C_{1}, C_{2}>0$. Since $\xi\left(x_{0}, \cdot\right)$ is locally integrable we conclude that $1-$ $2 \xi\left(x_{0}, \cdot\right) \in L_{1}(E, \infty)$ if and only if $\operatorname{Re} \widetilde{q}\left(x_{0}, \cdot\right) \in L_{1}\left(\mathbb{R}_{+}\right)$, which is equivalent to (2.5) due to

$$
\operatorname{Re} \widetilde{q}\left(x_{0}, t\right)=\int_{\mathbb{R}} q(x) \cos \sqrt{t}\left(x-x_{0}\right) d x .
$$

By Lebesgue's dominated convergence theorem we can pass to the limit in (2.7), and (2.6) is proven.

Let us establish the sufficient condition for (2.5). In the distributional sense

$$
\int_{\mathbb{R}} q(x) \cos t\left(x-x_{0}\right) d x=-\frac{1}{t} \int_{\mathbb{R}} q^{\prime}(x) \sin t\left(x_{0}-x\right) d x .
$$

Apply to the right-hand side of (2.9) the Schwartz inequality $(a>0)$

$$
\begin{aligned}
&\left(\int_{a}^{\infty}\right.\left.\left|\frac{1}{t} \int_{\mathbb{R}} q^{\prime}(x) \sin t\left(x-x_{0}\right) d x\right| d t\right)^{2} \\
& \quad \leq \int_{a}^{\infty} \frac{d t}{t^{2}} \cdot \int_{a}^{\infty}\left|\int_{\mathbb{R}} q^{\prime}(x) \sin t\left(x-x_{0}\right) d x\right|^{2} d t \\
& \quad \leq \frac{1}{a}\left\|\int_{\mathbb{R}} e^{i t x} q^{\prime}(x) d x\right\|_{2}^{2}=\frac{2 \pi}{a}\left\|q^{\prime}\right\|_{2}^{2},
\end{aligned}
$$

which is finite if $q \in H^{1,1}(\mathbb{R}) \cap H^{1,2}(\mathbb{R})$ and $(2.5)$ then holds. 
Remark 2.5. Theorem 2.4 was a concern of the recent paper [10 by Gesztesy, Holden, and Simon where it was proven under the condition $q \in H^{2,1}(\mathbb{R})$. In [16] we improved their result by showing that (2.5) is sufficient also conjecturing its necessity. Theorem 2.4 in its present form can be considered final.

Remark 2.6. Condition (2.5) forces $q$ to be continuous on $\left(-\infty, x_{0}\right)$ and $\left(x_{0}, \infty\right)$ [20] but not necessarily at $x_{0}$, i.e. $q\left(x_{0}-0\right)=q\left(x_{0}+0\right)$ need not hold. Note also that $q \in H^{1,1}(\mathbb{R})$ does not imply (2.5). Indeed, applying the Riemann-Lebesgue lemma to the right-hand side of (2.9) gives

$$
\int_{\mathbb{R}} q(x) \cos t\left(x-x_{0}\right) d x=o\left(\frac{1}{t}\right), t \rightarrow \infty,
$$

which only guarantees the so-called $A$-integrability of $1-2 \xi\left(x_{0}, \cdot\right)$ (see, e.g. [14] for the background information).

\section{Full-Line case with integrable potentials}

As opposed to the previous section if a priori $q \in L_{1}(\mathbb{R})$, then the pair $\left(H, H_{x_{0}}\right)$ defined by (2.1)-(2.3) is no longer our best choice. It is more natural to deal with $\left(H, H_{0}\right)$ :

$$
\begin{gathered}
H u=l u, u \in \operatorname{Dom}(H)=\left\{f \in L_{2}(\mathbb{R}): l f \in L_{2}(\mathbb{R})\right\}, \\
H_{0} u=-u^{\prime \prime}, u \in \operatorname{Dom}\left(H_{0}\right)=\left\{f \in L_{2}(\mathbb{R}): f^{\prime \prime} \in L_{2}(\mathbb{R})\right\} .
\end{gathered}
$$

Since $(H-i)^{-1}-\left(H_{0}-i\right)^{-1}$ is trace class, the spectral shift function $\xi(t)$ of $\left(H, H_{0}\right)$ exists. Our study of $\xi(t)$ will be based upon the concept of the Jost solution. We recall that the Jost solution $f(x, k)$ is the solution to the problem $(k \in \mathbb{R})$

$$
\left\{\begin{array}{l}
-u^{\prime \prime}+q(x) u=k^{2} u, x \in \mathbb{R}, \\
\lim e^{-i k x} u(x)=1, x \rightarrow \infty .
\end{array}\right.
$$

It is well-known that if $q \in L_{1}(\mathbb{R})$, then the Jost solution exists and is unique. The following statement follows from [17] and will play a crucial role in our consideration.

Proposition 3.1. Let $q \in L_{1}(\mathbb{R})$ and $f(x, k)$ be the Jost solution. Then $\forall k \in$ $\overline{\mathbb{C}}_{+} \backslash\left\{|k| \leq\|q\|_{1}\right\}$

$$
\begin{gathered}
e^{-i k x} f(x, k)=1+\int_{x}^{\infty} \frac{e^{2 i k(s-x)}-1}{2 i k} q(s) d s+\frac{1}{2}\left(\frac{1}{2 i k} \int_{x}^{\infty} q(s) d s\right)^{2}+r(x, k), \\
\text { where }\|r(\cdot, k)\|_{\infty}=O\left(|k|^{-2}\right) \text { and } r(x, k)=o\left(|k|^{-2}\right), \forall x \in \mathbb{R}, k \rightarrow \infty, k \in \overline{\mathbb{C}}_{+} .
\end{gathered}
$$

It is well-know that

$$
f(x, k)=T^{-1}(k)\left(e^{i k x}+R(k) e^{-i k x}\right)+o(1), x \rightarrow-\infty,
$$

where $T(k), R(k)$ denote the transmission and reflection coefficients. For $T(k)$ we have the following representation (see, e.g. [8]):

$$
T^{-1}(k)=1-\frac{1}{2 i k} \int_{\mathbb{R}} e^{-i k x} f(x, k) q(x) d x .
$$

The spectral shift function $\xi(t)$ of $\left(H, H_{0}\right)$ is related to $T$ (see, e.g. 2] by

$$
T^{-1}(\sqrt{\lambda})=\exp \left\{\int_{\mathbb{R}} \frac{\xi(t) d t}{t-\lambda}\right\} .
$$


We recall that $\sigma\left(H_{0}\right)=\sigma_{a c}\left(H_{0}\right)=\mathbb{R}_{+}$, the discrete spectrum $\sigma_{d}(H)=\left\{\lambda_{n}\right\}$ is negative, and $\left\{\lambda_{n}\right\}$ accumulates to at most $0, \sigma_{a c}(H)=\sigma_{a c}\left(H_{0}\right)=\mathbb{R}_{+}$and $\sigma(H)=\sigma_{d}(H) \cup \sigma_{a c}(H)$.

Our main result of this section is

Theorem 3.2. Let $q \in L_{1}(\mathbb{R}), \xi$ be the spectral shift function of $\left(H, H_{0}\right)$, and $\left\{\lambda_{n}\right\}=\sigma_{d}(H)$. Then $\left(Q=\int_{\mathbb{R}} q(x) d x\right)$

$$
\sum \lambda_{n}+\lim _{\varepsilon \rightarrow 0} \int_{0}^{\infty} \frac{1}{1+\varepsilon t}\left\{\xi(t)-\frac{Q}{2 \pi \sqrt{t}}\right\} d t=-\frac{Q^{2}}{4} .
$$

Relation (3.4) is absolutely summable if and only if

$$
\int_{0}^{\infty}\left(\int_{\mathbb{R}} q(s+x) q(s) d s\right) \sin t x d x \in L_{1}\left(\mathbb{R}_{+}, \frac{d t}{1+t}\right) .
$$

Formula (3.4) then reads

$$
\sum \lambda_{n}+\int_{0}^{\infty}\left\{\xi(t)-\frac{Q}{2 \pi \sqrt{t}}\right\} d t=-\frac{Q^{2}}{4} .
$$

Condition (3.5) holds if $q \in L_{1}(\mathbb{R}) \cap L_{2}(\mathbb{R})$.

Proof. By plugging (3.1) into (3.2) one has

$$
\begin{gathered}
T^{-1}(k)=1-\frac{Q}{2 i k}-\frac{1}{2 i k} \int_{\mathbb{R}} \int_{x}^{\infty}\left(\frac{e^{i k(s-x)}-1}{2 i k} q(s) d s\right) q(x) d x \\
-\frac{1}{2}\left(\frac{1}{2 i k}\right)^{3} \int_{\mathbb{R}}\left(\int_{x}^{\infty} q(s) d s\right)^{2} d x-\frac{1}{2 i k} \int_{\mathbb{R}} q(x) r(x, k) d x \\
=1+\frac{i Q}{2 k}+\left(\frac{1}{2 i k}\right)^{2}\left\{\frac{Q^{2}}{2}-\int_{0}^{\infty} e^{2 i k x}\left(\int_{\mathbb{R}} q(s+x) q(s) d s\right) d x\right\}+O\left(\frac{1}{k^{3}}\right),
\end{gathered}
$$

and hence, choosing the appropriate branch of the logarithm,

$$
\begin{aligned}
\ln T^{-1}(k) & =-\frac{Q}{2 i k}+\frac{1}{4 k^{2}}\left\{Q^{2}+\int_{0}^{\infty} e^{2 i k x}\left(\int_{\mathbb{R}} q(s+x) q(s) d s\right) d x\right\} \\
& +O\left(\frac{1}{k^{3}}\right) .
\end{aligned}
$$

Combining (3.3) and (3.7) yields

$$
\begin{aligned}
\int_{\mathbb{R}} \frac{\xi(t) d t}{t-\lambda} & =-\frac{Q}{2 i \sqrt{\lambda}} \\
& +\frac{1}{4 \lambda}\left\{Q^{2}+\int_{0}^{\infty} e^{2 i \sqrt{\lambda} x}\left(\int_{\mathbb{R}} q(s+x) q(s) d s\right) d x\right\}+O\left(\frac{1}{\lambda^{3 / 2}}\right) .
\end{aligned}
$$

One easily verifies that

$$
-\frac{1}{2 i \sqrt{\lambda}}=\frac{1}{2 \pi} \int_{0}^{\infty} \frac{1}{t-\lambda} \frac{d t}{\sqrt{t}}, \lambda \in \mathbb{C}_{+},
$$


and (3.8) obviously transforms into

$$
\begin{aligned}
& \int_{-\infty}^{0} \frac{\xi(t) d t}{t-\lambda}+\int_{0}^{\infty} \frac{1}{t-\lambda}\left\{\xi(t)-\frac{Q}{2 \pi \sqrt{t}}\right\} d t \\
& =\frac{1}{4 \lambda}\left\{Q^{2}+\int_{0}^{\infty} e^{2 i \sqrt{\lambda} x}\left(\int_{\mathbb{R}} q(s+x) q(s) d s\right) d x\right\}+O\left(\frac{1}{\lambda^{3 / 2}}\right) .
\end{aligned}
$$

Since [2] $\xi(t)=-N(t), t<0$, where $N(t)$ is the number of points of the discrete spectrum to the left from $t$, multiplying through by $\lambda$ one has

$$
\begin{aligned}
& -\int_{-\infty}^{0} \frac{\lambda}{t-\lambda} N(t) d t+\int_{0}^{\infty} \frac{\lambda}{t-\lambda}\left\{\xi(t)-\frac{Q}{2 \pi \sqrt{t}}\right\} d t \\
& =\frac{Q^{2}}{4}+\frac{1}{4} \int_{0}^{\infty} e^{2 i \sqrt{\lambda} x}\left(\int_{\mathbb{R}} q(s+x) q(s) d s\right) d x+O\left(\frac{1}{\sqrt{\lambda}}\right), \lambda \rightarrow \infty,
\end{aligned}
$$

uniformly in $\operatorname{Im} \sqrt{\lambda} \geq 0$. Due to the obvious estimate

$$
\left\|\int_{\mathbb{R}} q(s+x) q(s) d s\right\|_{1} \leq\|q\|_{1}^{2}
$$

the Riemann-Lebesgue lemma lets one pass in (3.10) to the limit

$$
-\lim _{\lambda \rightarrow-\infty} \int_{-\infty}^{0} \frac{\lambda}{t-\lambda} N(t) d t+\lim _{\lambda \rightarrow-\infty} \int_{0}^{\infty} \frac{\lambda}{t-\lambda}\left\{\xi(t)-\frac{Q}{2 \pi \sqrt{t}}\right\} d t=-\frac{Q^{2}}{4}
$$

Observing that by Lebesgue's dominated convergence theorem

$$
-\lim _{\lambda \rightarrow-\infty} \int_{-\infty}^{0} \frac{\lambda}{t-\lambda} N(t) d t=\int_{-\infty}^{0} N(t) d t=\sum_{n \geq 1} \lambda_{n}
$$

and setting $\lambda=-1 / \varepsilon$ we derive (3.4).

We now prove the second part of the theorem. It follows from (3.3) and (3.8) that

$$
\begin{aligned}
\xi(t) & =\frac{1}{\pi} \operatorname{Im} \ln T^{-1}(\sqrt{t}) \\
& =\frac{Q}{2 \pi \sqrt{t}}+\frac{1}{4 \pi t} \int_{0}^{\infty}\left(\int_{\mathbb{R}} q(s+x) q(s) d s\right) \sin 2 \sqrt{t} x d x+O\left(\frac{1}{t^{3 / 2}}\right) .
\end{aligned}
$$

Since $\xi$ is locally summable we conclude from (3.12) that $\xi(t)-\frac{Q}{2 \pi \sqrt{t}} \in L_{1}\left(\mathbb{R}_{+}\right)$if and only if

$$
\frac{1}{t} \int_{0}^{\infty}\left(\int_{\mathbb{R}} q(s+x) q(s) d s\right) \sin 2 \sqrt{t} x d x \in L_{1}(a, \infty), a>0,
$$

which is clearly equivalent to (3.5). Now by Lebesgue's dominated convergence theorem (3.4) implies (3.6).

Sufficiency of $q \in L_{1}(\mathbb{R}) \cap L_{2}(\mathbb{R})$ for (3.5) to hold immediately follows from

$$
\begin{aligned}
\left(\int_{a}^{\infty}\left|\frac{1}{t} \int_{\mathbb{R}} q(x) \sin t x d x\right| d t\right)^{2} & \leq \int_{a}^{\infty} \frac{d t}{t^{2}} \cdot \int_{a}^{\infty}\left|\int_{\mathbb{R}} q(x) \sin t x d x\right|^{2} d t \\
& \leq \frac{1}{a}\left\|\int_{\mathbb{R}} e^{i t x} q(x) d x\right\|_{2}^{2}=\frac{2 \pi}{a}\|q\|_{2}^{2}<\infty
\end{aligned}
$$

The theorem is proved. 
Remark 3.3. Relation (3.6) and its higher order analogs complement the chain of the so-called trace formulas first obtained by Faddeev-Zakharov [5] (see also [8]). The first of the Faddeev-Zakharov trace relations reads

$$
-\sum\left|\lambda_{n}\right|^{1 / 2}+\frac{1}{\pi} \int_{0}^{\infty} \ln |T(t)|^{-1} d t=\frac{Q}{4}
$$

which is related to the Lieb-Thirring estimates on $\sum\left|\lambda_{n}\right|^{1 / 2}$. Until recently it was an open question [8] whether (3.13) actually holds under the only condition $q \in$ $L_{1}(\mathbb{R})$. The affirmative answer was given by Weidl in 21]. Our relation (3.4) can be viewed as the second trace formula. Theorem 3.2 says that $q \in L_{1}(\mathbb{R})$ is not sufficient to derive even the second absolutely summable trace relation (3.6).

Remark 3.4. By the Riemann-Lebesgue lemma

$$
\frac{1}{t} \int_{0}^{\infty}\left(\int_{\mathbb{R}} q(s+x) q(s) d s\right) \sin t x d x=o\left(\frac{1}{t}\right), t \rightarrow \infty .
$$

It is not difficult to prove that (3.13) implies the $A$-integrability of $\xi(t)-\frac{Q}{2 \pi \sqrt{t}}$ and the resolvent regularization in (3.4) can be substituted for $(A) \int_{0}^{\infty}\left(\xi(t)-\frac{Q}{2 \pi \sqrt{t}}\right) d t$ (see also Remark 2.6). We plan to discuss it in detail in [18].

\section{HALF-Line CASE}

As the title of this section suggests we consider a pair of selfadjoint operators $\left(H_{x_{0}}^{+}, H_{0, x_{0}}^{+}\right)$defined as follows:

$$
\begin{gathered}
H_{x_{0}}^{+} u=l u, u \in \operatorname{Dom}\left(H_{x_{0}}^{+}\right)=\left\{f \in L_{2}\left(x_{0}, \infty\right): l f \in L_{2}\left(x_{0}, \infty\right), f\left(x_{0}+0\right)=0\right\}, \\
H_{0, x_{0}}^{+} u=-u^{\prime \prime}, u \in \operatorname{Dom}\left(H_{0, x_{0}}^{+}\right)=\left\{f \in L_{2}\left(x_{0}, \infty\right): f^{\prime \prime} \in L_{2}\left(x_{0}, \infty\right), f\left(x_{0}+0\right)=0\right\} .
\end{gathered}
$$

Of course it is only essential to treat the case when $x_{0}=0$, but there is a point in investigating the actual dependence on $x_{0}$.

Through this section we assume $q \in L_{1}(\mathbb{R})$. Then, as in the previous section, the spectral shift function $\xi^{+}\left(x_{0}, t\right)$ of $\left(H_{x_{0}}^{+}, H_{0, x_{0}}^{+}\right)$exists [13]. We recall that the spectrum of $H_{x_{0}}^{+}\left(H_{0, x_{0}}^{+}\right)$has a similar structure with $\sigma(H)\left(\sigma\left(H_{0}\right)\right)$ of section 3 .

Theorem 4.1. Let $q \in L_{1}(\mathbb{R}), \xi^{+}\left(x_{0}, \cdot\right)$ be the spectral shift function of the operators $\left(H_{x_{0}}^{+}, H_{0, x_{0}}^{+}\right)$, and $\left\{\lambda_{n}\left(x_{0}\right)\right\}=\sigma_{d}\left(H_{x_{0}}^{+}\right)$. Then for almost all $x_{0} \in \mathbb{R}$

$$
\sum \lambda_{n}\left(x_{0}\right)+\lim _{\varepsilon \rightarrow 0} \int_{0}^{\infty} \frac{1}{1+\varepsilon t}\left\{\xi^{+}\left(x_{0}, t\right)-\frac{Q\left(x_{0}\right)}{2 \pi \sqrt{t}}\right\} d t=-\frac{q\left(x_{0}\right)}{4},
$$

where $Q\left(x_{0}\right)=\int_{x_{0}}^{\infty} q(x) d x$. (4.1) is absolutely summable if and only if

$$
\int_{x_{0}}^{\infty} q(x) \cos t\left(x-x_{0}\right) d x \in L_{1}\left(\mathbb{R}_{+}, d t\right) .
$$

Formula (4.1) then reads: for all $x_{0} \in \mathbb{R}$

$$
\sum \lambda_{n}\left(x_{0}\right)+\int_{0}^{\infty}\left\{\xi^{+}\left(x_{0}, t\right)-\frac{Q\left(x_{0}\right)}{2 \pi \sqrt{t}}\right\} d t=-\frac{q\left(x_{0}\right)}{4} .
$$

Condition (4.2) holds if $q \in H^{1,1}\left(x_{0}, \infty\right) \cap H^{1,2}\left(x_{0}, \infty\right)$. 
Proof. It is enough to prove the theorem for the case of $x_{0}=0$. Set $\lambda_{n}(0)=$ $\lambda_{n}, \xi^{+}(0, t)=\xi^{+}(t), Q(0)=Q$. Our prove is based upon the relation (see, e.g. [2])

$$
f(0, \sqrt{\lambda})=\exp \left\{\int_{\mathbb{R}} \frac{\xi^{+}(t)}{t-\lambda} d t\right\}
$$

where $f(x, k)$ is the Jost solution. It follows from (4.4) and Proposition 3.1 that

$$
\int_{\mathbb{R}} \frac{\xi^{+}(t)}{t-\lambda} d t=\ln f(0, \sqrt{\lambda})=-\frac{Q}{2 i \sqrt{\lambda}}+\frac{1}{2 i \sqrt{\lambda}} \int_{0}^{\infty} e^{2 i \sqrt{\lambda}(x)} q(x) d x+o\left(\frac{1}{\lambda}\right) .
$$

Repeating the arguments of Theorem 3.2, (4.5) transforms into

$$
\begin{gathered}
\int_{-\infty}^{0} \frac{\xi^{+}(t) d t}{t-\lambda}+\int_{0}^{\infty} \frac{1}{t-\lambda}\left\{\xi^{+}(t)-\frac{Q}{2 \pi \sqrt{t}}\right\} d t \\
=\frac{1}{2 i \sqrt{\lambda}} \int_{0}^{\infty} e^{2 i \sqrt{\lambda} x} q(x) d x+o\left(\frac{1}{\lambda}\right)
\end{gathered}
$$

which in turn implies

$$
\begin{aligned}
& -\int_{-\infty}^{0} \frac{\lambda}{t-\lambda} N(t) d t+\int_{0}^{\infty} \frac{\lambda}{t-\lambda}\left\{\xi^{+}(t)-\frac{Q}{2 \pi \sqrt{t}}\right\} d t \\
& =\frac{\sqrt{\lambda}}{2 i} \int_{0}^{\infty} e^{2 i \sqrt{\lambda} x} q(x) d x+o(1), \lambda \rightarrow \infty,
\end{aligned}
$$

where $N(t)$ as in Theorem 3.2. Choosing in (4.6) $\sqrt{\lambda}=i \tau, \tau>0$, and letting $\tau \rightarrow \infty$, we obtain

$$
\begin{aligned}
& \lim _{\tau \rightarrow \infty} \int_{-\infty}^{0} \frac{\tau}{t+\tau} N(t) d t+\lim _{\tau \rightarrow \infty} \int_{0}^{\infty} \frac{-\tau}{t+\tau}\left\{\xi(t)-\frac{Q}{2 \pi \sqrt{t}}\right\} d t \\
& =\lim _{\tau \rightarrow \infty} \frac{\tau}{2} \int_{0}^{\infty} e^{-2 \tau x} q(x) d x .
\end{aligned}
$$

By Lemma 3.2 from [16] we have

$$
\lim _{\tau \rightarrow \infty} \frac{\tau}{2} \int_{0}^{\infty} e^{-2 \tau x} q(x) d x=\frac{q(0)}{4},
$$

provided that 0 is a right Lebesgue point of $q$. We are reminded that a point $x$ is called a right (left) Lebesgue continuity point of a function $f(x) \in L_{1}$ if (see, e.g. 20])

$$
\int_{0}^{h}|f(x \pm t)-f(x)| d t=o(h), h \rightarrow+0 .
$$

The limits on the left-hand side of (4.7) are computed in the same manner as in Theorem 3.2. Combining (4.7), (4.8) and setting $\varepsilon=1 / \tau$ we have

$$
\sum \lambda_{n}+\lim _{\varepsilon \rightarrow 0} \int_{0}^{\infty} \frac{1}{1+\varepsilon t}\left\{\xi^{+}(t)-\frac{Q}{2 \pi \sqrt{t}}\right\} d t=-\frac{q(0)}{4} .
$$

Returning now to the general right Lebesgue point $x_{0} \in \mathbb{R}$ of $q(x)$ we finally obtain (4.1). But almost all points of a summable function are Lebesgue! 
We use the same arguments as in Theorem 2.4 to derive that $\xi(t)-\frac{Q}{2 \pi \sqrt{t}} \in$ $L_{1}\left(\mathbb{R}_{+}\right)$if and only if

$$
\frac{1}{\sqrt{t}} \int_{0}^{\infty} q(x) \cos \sqrt{t} x d x \in L_{1}\left(\mathbb{R}_{+} ; d t\right)
$$

that is equivalent to (4.2) in case of an arbitrary $x_{0}$. Observe now that condition (4.2) forces $q(x)$ to be continuous on $\left[x_{0}, \infty\right)$ and hence under (4.2) the right-hand side of (4.1) is defined for all $x_{0}$. Applying Lebesgue's dominated convergence we arrive at (4.3). Sufficiency of the condition $q \in H^{1,1}\left(x_{0}, \infty\right) \cap H^{1,2}\left(x_{0}, \infty\right)$ should also be clear in virtue of the similar arguments of Theorem 2.4.

Remark 4.2. Relation (4.1) is the second formula (the first one is similar to (3.13)) in the chain of the trace formulas obtained (in the case of $x_{0}=0$ ) by Buslaev and Faddeev in [3] under excessively strong conditions on $q$. Theorem 4.1 shows that even though the condition $q \in L_{1}(\mathbb{R})$ is enough for the existence of $\xi^{+}\left(x_{0}, t\right) \in$

$L_{1}\left(\mathbb{R} ; \frac{d t}{1+t^{2}}\right)$ it does not imply (4.3), i.e. absolute summability of (4.1). Note however that as in section 3 the first trace formula does exist.

\section{REFERENCES}

1. F. Atkinson, "On the location of the Weyl circles", Proc. Roy. Soc. Edinburgh 88A (1981), 345-356.

2. M.Sh. Birman and D.R. Yafaev, "The spectral shift function. The work of M.G. Krein and its further development", St. Petersburg Math. J. 4 (1993), 833-870. MR 94g:47002

3. V.S. Buslaev and L.D. Faddeev, "Formulas for traces for a singular Sturm-Liouville differential operator", Soviet Math. Dokl. 1 (1960), 451-454. MR 22:11171

4. L.A. Dikii, "Trace formulas for Sturm-Liouville differential operators", Am. Math. Soc. Trans. 18 (1961), 81-115. MR 23:A1874

5. L.D. Faddeev and V.E. Zakharov, "The Korteweg-De Vries equation is a fully integrable Hamiltonian system", Funct. Anal. Appl., 5 (1971), 18-27. MR 46:2270

6. I.M. Gel'fand and B.M. Levitan, "On a simple identity for eigenvalues of a second order differential operator", Dokl. Akad. Nauk SSSR 88 (1953), 593-596 (in Russian); English translation in I.M. Gel'fand, Collected Papers (Springer Verlag, Berlin, 1987), Vol. 1, 457-461. MR 15:33a

7. F. Gesztesy, H. Holden, B. Simon, and Z. Zhao, "Trace formulae and inverse spectral theory for Schrödinger operators", Bull. Am. Math. Soc. 29 (1993), 250-255. MR 94c:34127]

8. F. Gesztesy and H. Holden, "Trace formulas and conservation laws for nonlinear evolution equation", Rev. Math. Phys. 6, 1 (1994), 51-95. MR 95h:35198a errata MR 95h:35198b

9. F. Gesztesy, H. Holden, B. Simon, and Z. Zhao, "Higher order trace relations for Schrödinger operators", Rev. Math. Phys. 7, 6 (1995), 893-922. MR 97d:34094

10. F. Gesztesy, H. Holden, and B. Simon, "Absolute summability of the trace relation for certain Schrödinger operators", Commun. Math. Phys. 168 (1995), 137-161. MR 96b:34110

11. F. Gesztesy and B. Simon, "The $\xi$ function", Acta Math. 176 (1996), 49-71. MR 97e:47078

12. F. Gesztesy and B. Simon, "A new approach to inverse spectral theory, II. General real potentials and the connection to the spectral measure", Ann. Math. 152 (2000), 593-643. MR 2001m:34185b

13. V.A. Javrjan, "The spectral shift function for Sturm-Liouville differential operators", Akad. Nauk Armjan. SSR Dokl 38 (1964), 193-198. MR 29:3710

14. A.V. Rybkin, "On $A$-integrability of the spectral shift function of unitary operators arising in the Lax-Phillips scattering theory", Duke Math. J. 83 (1996), 683-699. MR 97e:58224

15. A.V. Rybkin "KdV invariants and Herglotz functions", Differential Integral Equations, 14, no. 4 (2001), 493-512. MR 2001k:34155

16. A.V. Rybkin, "On the trace approach to the inverse scattering in dimension one", SIAM J. Math. Anal. 32 (2001), 6, 1248-1264.

17. A.V. Rybkin, "Some new and old asymptotic representations of the Jost solution and Weyl $m$-function for Schrödinger operators on the line", Bulletin of London Math Soc. 34 (2002), 61-72. CMP 2002:04 
18. A.V. Rybkin, "A Weyl $m$-function approach to trace formulas for various Schrödinger operators", in preparation.

19. E.C. Titchmarsh, "On eigenfunction expansions associated with second-order differential equations" Oxford University Press 1946. MR 8:458d

20. E.C. Titchmarsh, "Introduction to the theory of Fourier integrals", Chelsea, New York, 1986. MR 89c: 42002

21. T. Weidl, "On the Lieb-Thirring constants $L_{\gamma, 1}$ for $\gamma \geq 1 / 2$ ", Commun. Math. Phys. 178 (1996), 135-146. MR 97c:81039

Department of Mathematical Sciences, University of Alaska, Fairbanks, P.O. Box 756660, FAirbanks, Alaska 99775

E-mail address: ffavr@uaf.edu 